\title{
DYNAMICS BETWEEN ORDER AND CHAOS IN A SIMPLE REENTRANT MODEL OF PRODUCTION DYNAMICS
}

\author{
I. KATZORKE and A. PIKOVSKY \\ Department of Physics, University of Potsdam, \\ Potsdam, Germany
}

Received January 3, 2002; Revised February 20, 2002

\begin{abstract}
We consider a simple reentrant model of a manufacturing process, consisting of one machine at which two different types of items have to be processed. The model is completely deterministic: all delivery and processing times are fixed, and are generally incommensurate. Dependent on the arrival and processing times, a queue of waiting items grows, remains constant or disappears. We demonstrate that the dynamics of the system crucially depends on the queue type. Complexity is most observed for the case of growing queue. We characterize this dynamics between order and chaos with the T-entropy and the autocorrelation function.
\end{abstract}

Keywords: Production dynamics; quasiperiodicity; complexity.

\section{Introduction}

In the queue theory one describes the operation of production systems supposing that the input orders are random in time (e.g. Poissonian processes) [Nelson, 1995; Gross \& Harris, 1985]. Thus, the queue theory is often considered as a part of the theory of stochastic processes. Recently, purely deterministic models of production systems have attracted a growing interest. In these models no external stochasticity is assumed, i.e. the times at which the orders appear are deterministic (in the simplest case periodic), see, e.g. [Bunimovich, 2001]. If the production process is also deterministic, one can assume that prediction and controlling of such a production system is relatively simple. However, theoretical [Chase et al., 1993] as well as empirical [Beaumariage \& Kempf, 1994] studies have shown that completely deterministic production systems can demonstrate complex behavior. This is not very surprising, because chaos is one of the possible types of dynamical behavior. The production systems have, however, some specific properties that make the appearance of chaos not so common as in general dynamical systems. In particular, usually one considers processing of discrete units (items), and chaos can disappear in course of discretization. One such example has been recently described in [Katzorke \& Pikovsky, 2000], where it has been shown that chaos in a continuous-state production system reduces to a complex but nonchaotic behavior if one considers processing of discrete items.

A particular interest in the studies of deterministic queue models have attracted so-called reentrant systems (see e.g. [Hanson et al., 1999]). A reentrant system models a manufacturing process, where the items to be processed have to pass one machine (or a group of machines) more than one time. In [Diaz-Rivera et al., 2000] an example of such a reentrant model, consisting of two machines with different scheduling policies for each machine, has been investigated. It has been demonstrated that depending on the ratio of the work velocities, either the periodic dynamics or a behavior between chaos and periodicity is observed.

In this paper we investigate a simple reentrant model where only one machine processes different 
types of items twice. The main parameters here are the arrival and the processing times, which are in general different. It turns out, that the observed behavior in this model strongly depends on the kind of the queue of waiting items and, in some cases, on the initial conditions.

This paper is organized as follows. In Sec. 2 the model is formulated. We describe the main relevant parameters and introduce the balance condition important for the dynamical properties. In Secs. 3 and 4 we study stationarity, ergodicity, and sensitivity to the initial conditions. In Secs. 5 and 6 complexity of the dynamics is characterized via autocorrelations and T-entropy. Some generalizations are discussed in the conclusion. In particular, we consider there an analogy of the model under consideration with traffic models.

\section{Basic Model}

\subsection{Reentrant model}

In this section we describe a simple reentrant model that we will investigate in this paper. In general, reentrance means that there is a single machine or a group of machines, which items have to pass the system for more than one time. A typical case of such organization one finds in the microelectronic chip production. In our simplest model there is just one machine, symbolized with a box in Fig. 1. Two different kinds of workpieces $A$ and $B$ arrive at this machine, and these items have to pass it twice. We denote the items after first processing as $A^{\prime}$ and $B^{\prime}$, respectively. All the items that have to enter the machine are organized in a queue according to the FIFO (first in-first out) rule (in the case when two

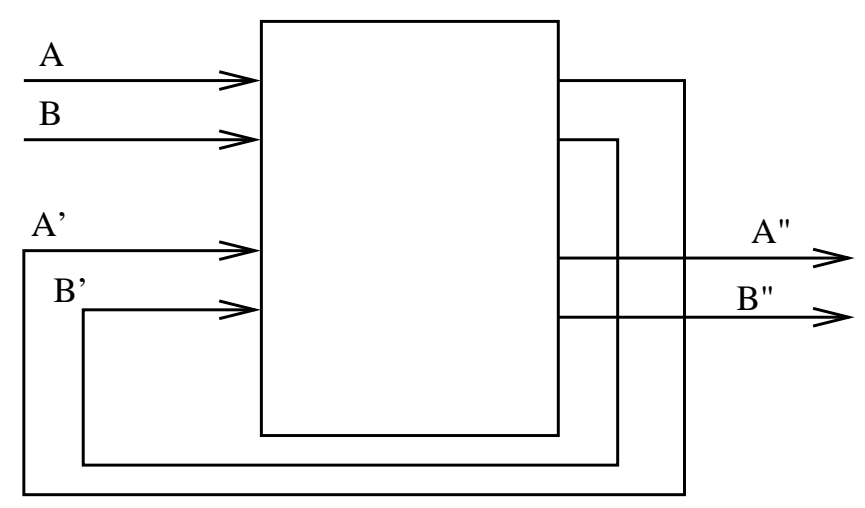

Fig. 1. A scheme of the item flow for the model described in text. Two different kinds of workpieces $A$ and $B$ have to pass the machine twice until they leave it as $A^{\prime \prime}$ and $B^{\prime \prime}$. items arrive simultaneously, the priority according to the ordering $A>B>A^{\prime}>B^{\prime}$ is used). This means that at the input of the machine we have a series of four symbols $A, B, A^{\prime}, B^{\prime}$ which is transformed at the output to the corresponding series of $A^{\prime}, B^{\prime}, A^{\prime \prime}, B^{\prime \prime}$. We assume that the items $A$ and $B$ are entering at deterministic periodic time intervals $t_{A}$ and $t_{B}$, respectively. Other relevant parameters are the processing times, i.e. the times needed for processing the items $A, B, A^{\prime}, B^{\prime}$. We denote these times as $T_{A}, T_{B}, T_{A^{\prime}}, T_{B^{\prime}}$, respectively. We assume that these times are constants, i.e. they are independent of the length of the queue, the order of the items in the queue, etc.

For the dynamics of the system it is essential, that the times $t_{A, B}, T_{A, B, A^{\prime}, B^{\prime}}$ are arbitrary real numbers. Thus, in general they are incommensurate (not in a rational relation). This makes the whole process at least as complex as quasiperiodic. We will see that in certain situations this quasiperiodicity of input is reproduced in the queue and in the output, while in other cases the complexity grows and the output is more complex than quasiperiodic.

Two types of output observables are possible. One can either observe the times at which the consequent pieces of work are accomplished, or one can observe the sequence of outcoming symbols. In this paper we will mainly use the second, symbolic characterization of the process. It allows us to apply different measures of complexity with the most efficiency. We note also, that the output sequence is equivalent to the sequence of symbols at the input of the machine.

\subsection{The balance condition}

Depending on its productivity (capacity), the machine will be able to accomplish all the incoming pieces of work, or not. In terms of the parameters of the system, i.e. of the times $t_{A, B}, T_{A, B, A^{\prime}, B^{\prime}}$, this can be formulated as follows. Let us consider a large time interval $T$. During this time $N_{A}=T / t_{A}$ items of type $A$ and $N_{B}=T / t_{B}$ items of type $B$ are delivered. The total processing time for these items is $N_{A}\left(T_{A}+T_{A^{\prime}}\right)+N_{B}\left(T_{B}+T_{B^{\prime}}\right)$. This time should be compared with the time interval $T$. Thus, the critical processing capacity is described by the parameter $\tau$ :

$$
\tau=\frac{T_{A}+T_{A^{\prime}}}{t_{A}}+\frac{T_{B}+T_{B^{\prime}}}{t_{B}}
$$

The following kinds of systems can be distinguished according to the value of $\tau$ : 
- $\tau<1$ : the underbalanced system, here the incoming item flow is less than the capacity;

- $\tau=1$ : the balanced system, here the incoming flow fits exactly the processing capacity;

- $\tau>1$ : the overbalanced system; here the processing capacity is insufficient to process all the incoming units.

According to this classification, the queue will disappear, remain, or grow depending on the value of $\tau$.

\subsection{The reentrant model as a dynamical system}

In this subsection we represent the reentrant model described above as a dynamical system. This dynamical system is rather unusual, because it has two types of the variables. The state of the queue is described by a symbolic sequence of symbols $A, B, A^{\prime}, B^{\prime}$. The current state of the machine is described by a continuous variable $x$ which can be viewed as the processing phase. The phase $x$ grows linearly in time:

$$
\frac{d x}{d t}=\frac{1}{T_{S}}
$$

where $S$ stands for the item which is in processing. As $x$ reaches the value 1 , the processing of the item ends, and the queue is rearranged: the first-in item enters the machine, and, in the case $S=A$ or $S=B$, the corresponding item $\left(A^{\prime}\right.$ or $\left.B^{\prime}\right)$ is added to the queue. Additionally, every time step $t_{A}$ and $t_{B}$ the items $A$ and $B$ are added to the queue, this latter operation can be considered as a quasiperiodic (in the general case of incommensurate $t_{A}$ and $t_{B}$ ) forcing of the system.

The dynamical system is completely described by the above rules. Its time-continuous part is the trivial linear equation (2). However, complex dynamics is not excluded. On one hand, complexity can appear due to "boundary conditions" to (2), as is the case for strange billiards [Chase et al., 1993; Schürmann \& Hoffmann, 1995]. On the other hand, the system is driven quasiperiodically, which means that the minimal possible complexity is that of a quasiperiodic process.

The parameters governing the dynamics are the times $t_{A}, t_{B}, T_{A}, T_{B}, T_{A^{\prime}}, T_{B^{\prime}}$. We have performed simulations with different sets of these parameters. Below, if the opposite is not explicitly stated, we use the following parameters: $t_{A}=1, t_{B}=1+\sqrt{5}, T_{A}=$ $T_{A^{\prime}}=(2 \sqrt{2})^{-1}, T_{B}=T_{B^{\prime}}=c^{-1}(2-\sqrt{2})(\sqrt{5}-1)^{-1}$.
Here the parameter $c$ governs the balance condition. From (1) one easily obtains $\tau=c^{-1}(1+(c-1) / \sqrt{2})$.

As the main observable providing us the information on the systems' dynamics we use the symbolic sequence of the machine input queue. For calculation of the correlation function we assign the numerical values to the symbols as follows: $A \rightarrow 1, B \rightarrow 2, A^{\prime} \rightarrow 3, B^{\prime} \rightarrow 4$. As a result, a time series $z_{i}, 1 \leq z \leq 4$ is produced.

\section{Sensitivity to Initial Conditions}

One of the main characterizations of the nonlinear dynamics is the sensitivity to initial conditions. In smooth dynamical systems one calculates the maximal Lyapunov exponent, which allows one to distinguish unstable (positive LE), neutral (zero LE) and stable (negative LE) dynamics. In our case of the hybrid phase space where a part of variables is symbolic, we cannot define the Lyapunov exponent, because an infinitesimal perturbation of the symbolic variable is not possible. Instead, we perform the following numerical experiment: we perturb slightly the dynamics governed by Eq. (2) and observe the changes in our symbolic observable - the queue.

The first observation is that any changes in the symbolic sequence appear first with a delay $\delta t_{1}$ after the perturbation is imposed. We can roughly estimate $\delta t_{1} \sim \Delta^{-1}$, where $\Delta$ is the perturbation strength. This is valid for all types of the system (overbalanced, balanced and underbalanced), and correspond to a simple observation that a small shift of the times of arrival of items $A^{\prime}$ and $B^{\prime}$ to the queue leads to a change in the symbolic sequence only when one of these arrival times is very close to the arrival time of one of the items $A, B$. Due to incommensurate relation between the characteristic times this always happens, and due to ergodicity of the incoming quasiperiodic sequence one has to wait for approximately $\Delta^{-1}$ until this happens.

In the case of a balanced and overbalanced systems the produced change in the symbolic sequence never disappears, because the queue is never empty and the nondisturbed arrival times cannot be restored. On the other hand, in the underbalanced case the queue is from time to time empty. In these periods the perturbation of the shifted arrival times disappears, and the unperturbed symbolic sequence is restored. Thus, the effect of perturbation on the underbalanced dynamics is temporary: it exists only during the time interval $\delta t_{1}<t<\delta t_{2}$ after the 


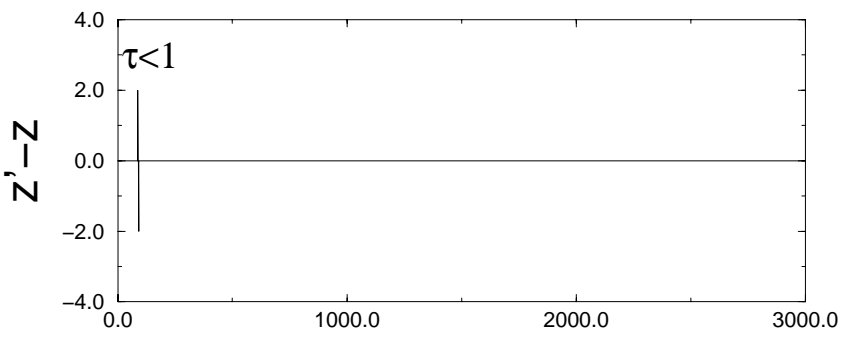

(a)

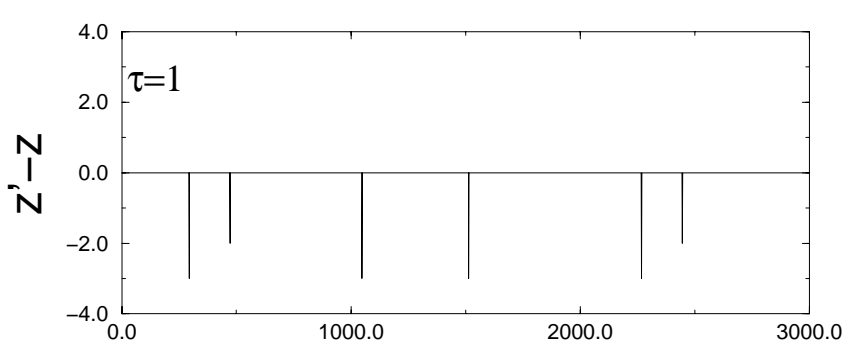

(b)

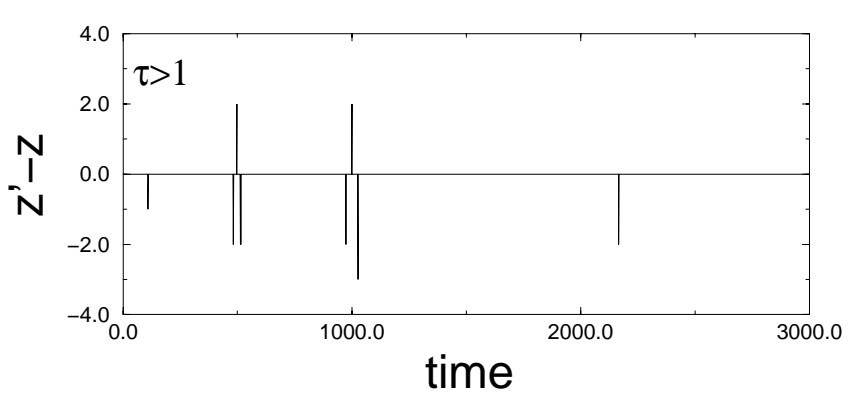

(c)

Fig. 2. The sensitivity of the symbolic sequences: the difference of the symbols of the perturbed (at timestep 4) and the unperturbed sequences. (a) Underbalanced system $c=1.1$, (b) balanced system $c=1$, (c) overbalanced system $c=0.9$. The perturbation is $c_{p}=c \cdot 1.001$ in all cases.

perturbation is imposed. Here $\delta t_{2}$ is the time at which the sum of all empty queue states exceeds the perturbation. Clearly, $\delta t_{2}$ is inversely proportional to $(1-\tau)$. We illustrate the dynamics of the perturbation in Fig. 2.

\section{Ergodicity and Stationarity}

In this section we focus on two features of the dynamics, which allow us to characterize the observed time series with suitable methods of the theory of stochastic processes. The first property means that the characteristics do not depend on initial conditions, i.e. some sort of "ergodicity" is valid. The second property - stationarity - means that there is no trend in statistical properties of the time series, which allows us to apply characteristics suitable for stationary signals.

\subsection{Ergodicity}

To check the ergodicity we looked at how the observed transition probabilities in a time series (i.e. probabilities to find pairs of symbols, like $A B^{\prime}$ ) depend on initial state. For $c \neq 1$ we have found that the transition probabilities do not depend on initial state, while for $c=1$ such a dependence is observed (Fig. 3). This means that in the case of perfect balance the dynamics is not ergodic and has at least one "integral of motion", while nonbalanced systems do not have such an integral.

Qualitatively, such a dependence on the balance condition can be understood as follows. In the underbalanced case the dynamics, as we have shown above, is not sensitive to the initial conditions, and is fully determined by the quasiperiodic drive (input flow of items). Therefore, the system is ergodic. In the overbalanced case, the queue grows, thus any perturbation in the dynamics increases because it affects more and more items in the queue. As a result, effective "averaging" over all initial perturbations occurs resulting in ergodic behavior. In the balanced case a perturbation in the initial conditions remains roughly constant in time course, because the queue length is constant. Thus, different initial conditions do not "mix" and the statistics can be dependent on them, thus breaking the ergodicity.

\subsection{Stationarity}

A stationarity of the observed symbolic time series is ensured in the underbalanced case, where the dynamics is essentially driven by the quasiperiodic input, and thus follows the stationarity of the quasiperiodic process. For other conditions this argument does not work. In particular, for the overbalanced case the stationarity may be doubted because the queue grows and this may lead to a trend in the statistical properties. Therefore we have tested the stationarity numerically. For this end we have produced a long symbolic time series (up to 700.000), divided it into several parts, and compared statistical characteristics of the sequence in these parts. Particularly, we estimated the probabilities of combinations of symbols (there are 16 possible combinations of two and 64 combinations of three symbols), and used the $\chi^{2}$-criterion to judge whether the hypothesis that these probabilities are 


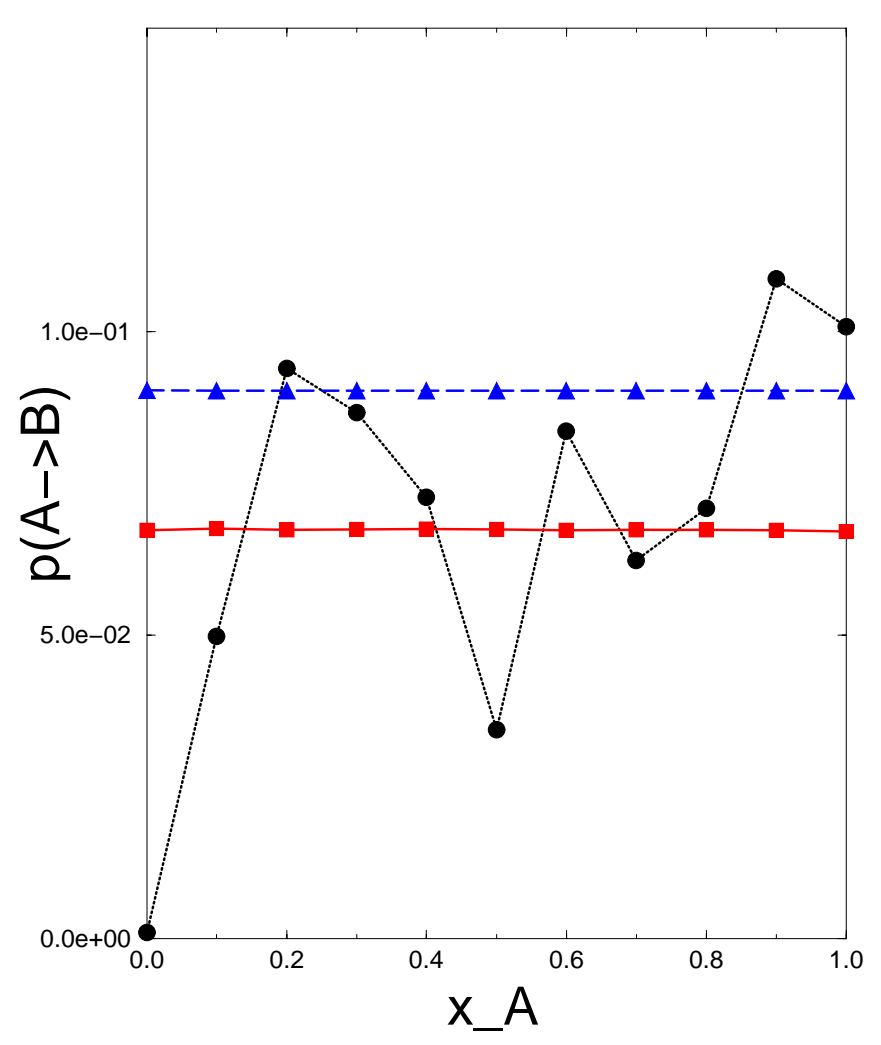

(a)

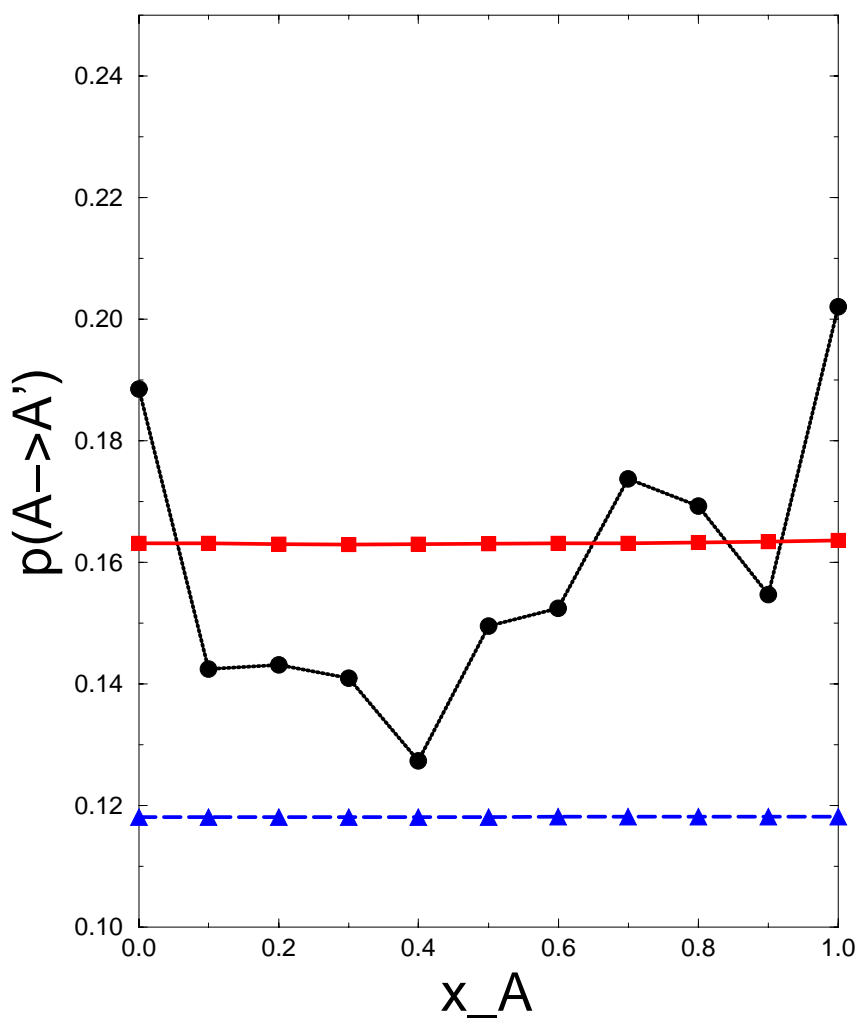

(b)

Fig. 3. The probability to find (a) a pair $(A, B)$ or (b) a pair $\left(A, A^{\prime}\right)$ in the queue is shown for the balanced (solid line, filled circles), the overbalanced (dotted line, filled squares) and for the underbalanced (dashed line, filled triangles) systems depending on the initial conditions $x_{A}$. Note that only the balanced system demonstrates dependence on initial conditions.

the same in different parts of the time series can be approved or should be rejected [Press et al., 1989]. For the values of the parameter $c$ not very close to 1 we could always confirm the stationarity hypothesis (Fig. 4). If $c$ is close to 1 (e.g. $c \approx 0.995$ ), we have difficulties in applying the test because the statistical properties appear to have very slow modulations, thus requiring averaging over extremely long time intervals. This will be also seen in characterization of the correlations in the next section.

\section{Correlations}

For the calculations of the normalized autocorrelation function we use the integer time series $z_{i}$ as defined above:

$$
C(k)=\frac{\left\langle z_{i+k} z_{i}\right\rangle-\langle z\rangle^{2}}{\left\langle z^{2}\right\rangle-\langle z\rangle^{2}} .
$$

In the underbalanced case the autocorrelation function demonstrates a typical for quasiperiodic dynamics pattern, returning nearly to 1 at a relatively regular rate (Fig. 5). This supports the conclusion above that in this case the behavior of the system is completely determined by the quasiperiodic driving.

In the case of perfect balance $(\tau=c=1)$ the dynamical behavior depends on initial conditions, so the autocorrelation function depends on them as well. We show two examples in Fig. 6. In all observed situations for some large time shifts $k$ the correlations are close to 1 , thus indicating the quasiperiodicity. We note that in some cases the correlations for small $k$ are not close to one, this means that on a small time scale the process is more complex than quasiperiodic.

The largest complexity is achieved in the overbalanced case. Here the autocorrelations do not return to 1 even for large time shifts $k$. Several examples of the autocorrelation function for $0.98 \leq$ $c \leq 0.999$ are presented in Fig. 7. This function looks on a small scale like a quasiperiodic one, but with a slowly varying envelope that is smaller than 1. This envelope is shown in Fig. 7 for different values of parameter $c$, i.e. for different levels of 


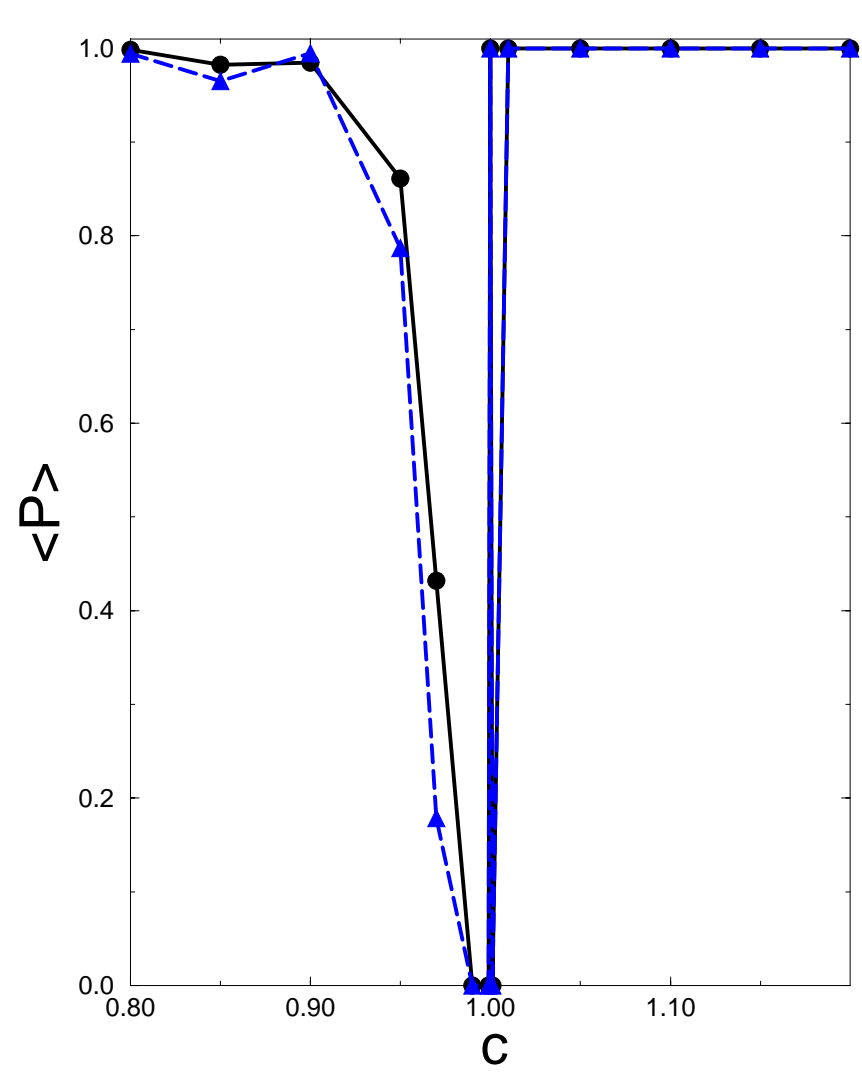

Fig. 4. Results of the application of the $\chi^{2}$-test to the stationarity of symbolic sequences. Large values of $p$ correspond to the acceptance of the stationarity hypothesis. Combinations consisting of two symbols (solid line, filled circles) and of three symbols (dashed line, triangles) have been analyzed.

violation of the balance condition. One can see that for larger deviations of $c$ from 1 the correlations decay faster, and to a lower level.

Because the correlations do not achieve level 1 , the process is more complex than quasiperiodic. We conjecture that the spectrum of the process has a singular continuous component [Queffélec, 1987]. Such spectra have been studied recently for complex nonchaotic systems like strange nonchaotic attractors [Pikovsky \& Feudel, 1994], substitution sequences [Zaks et al., 1998], fluid flows [Zaks, 2001], etc. Unfortunately, in the present case it is hardly possible to extract the singular continuous component from the spectrum, because it has also very strong discrete components.

\section{Complexity}

From the general point of view of the theory of complexity [Badii \& Politi, 1997] we face a problem of characterizing a complexity of a symbolic

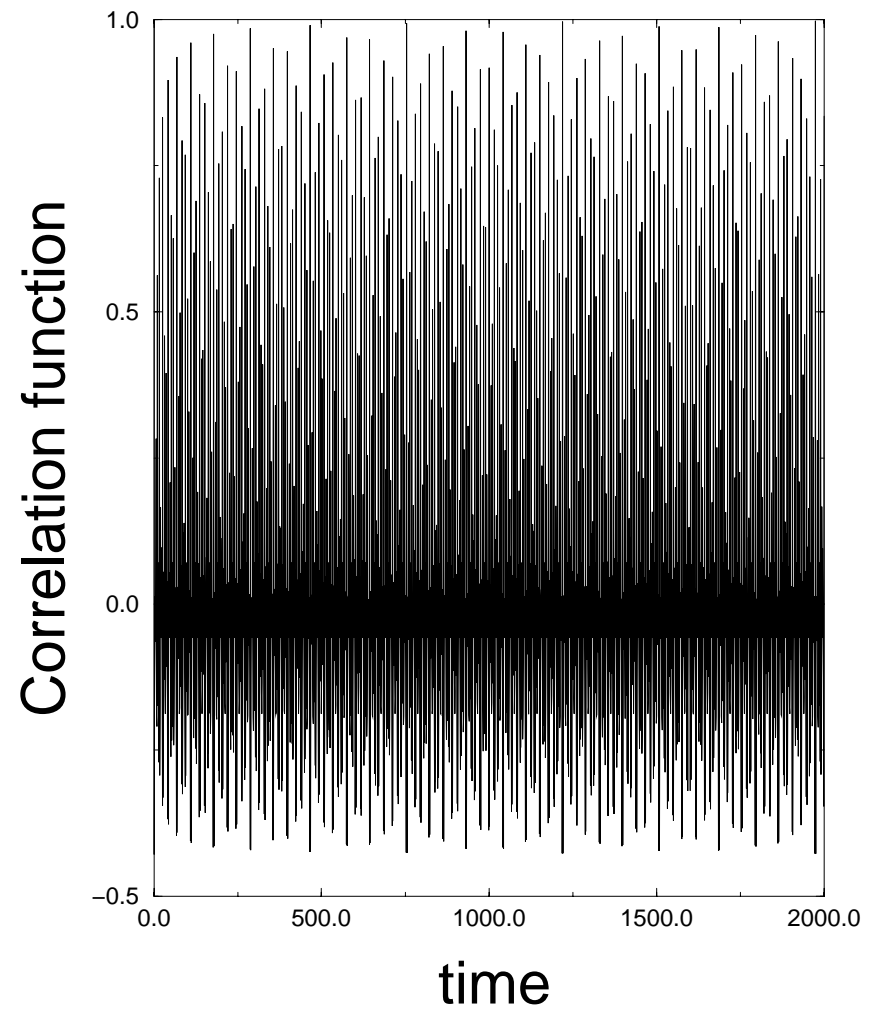

Fig. 5. Correlation function of underbalanced system $c=1.2$.

series of four symbols $A, B, A^{\prime}, B^{\prime}$. Here we use a recently suggested method by Titchener [1994, 1998, 2001].

This method consists in the recursive determination of single words or combinations of letters. The algorithm works as follows: First the last letter $k_{1}$ of the sequence has to be found, and how many times this symbol (the letter or sign) is found in the sequence is counted, starting from the end. If the next symbol $k_{n}$ is different from the previous symbol $k_{n-1}$, the number of identical stings at the step $n$ will be one and $n$ increases by one. Then the counting of the identical strings is repeated with the string consisting of the last two symbols of the sequence, then with the last three symbols and so forth. Thus the number $k_{n}$ increases by more than one only, if there are some repetitions of patterns, like $A \rightarrow B \rightarrow A \rightarrow B \rightarrow A \rightarrow B \ldots$ The number of repetitions is identical to the number of $k_{n}$. If the repetitions end, a new group of symbols will be used, as described above for the estimation of the next $k_{n+1}$. The new string starts at the end of the previous string (in this case the repetition) and will be built backwards, that means from the symbols $z$ to $z-i$. 


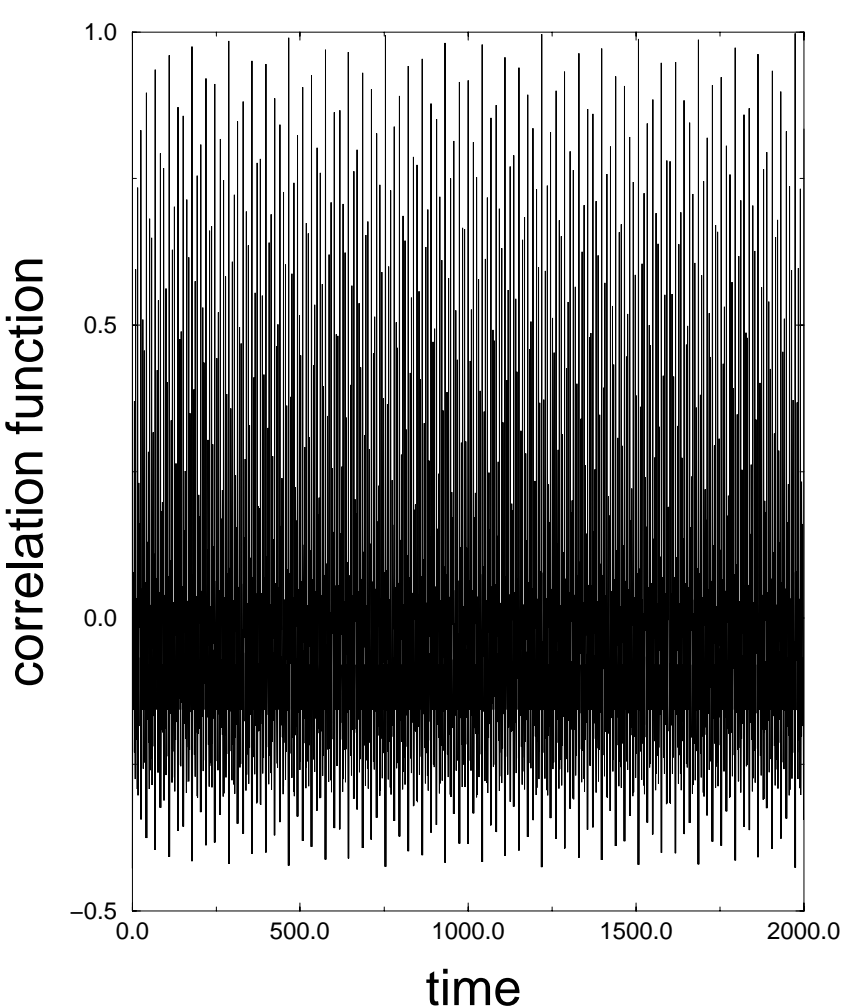

(a)

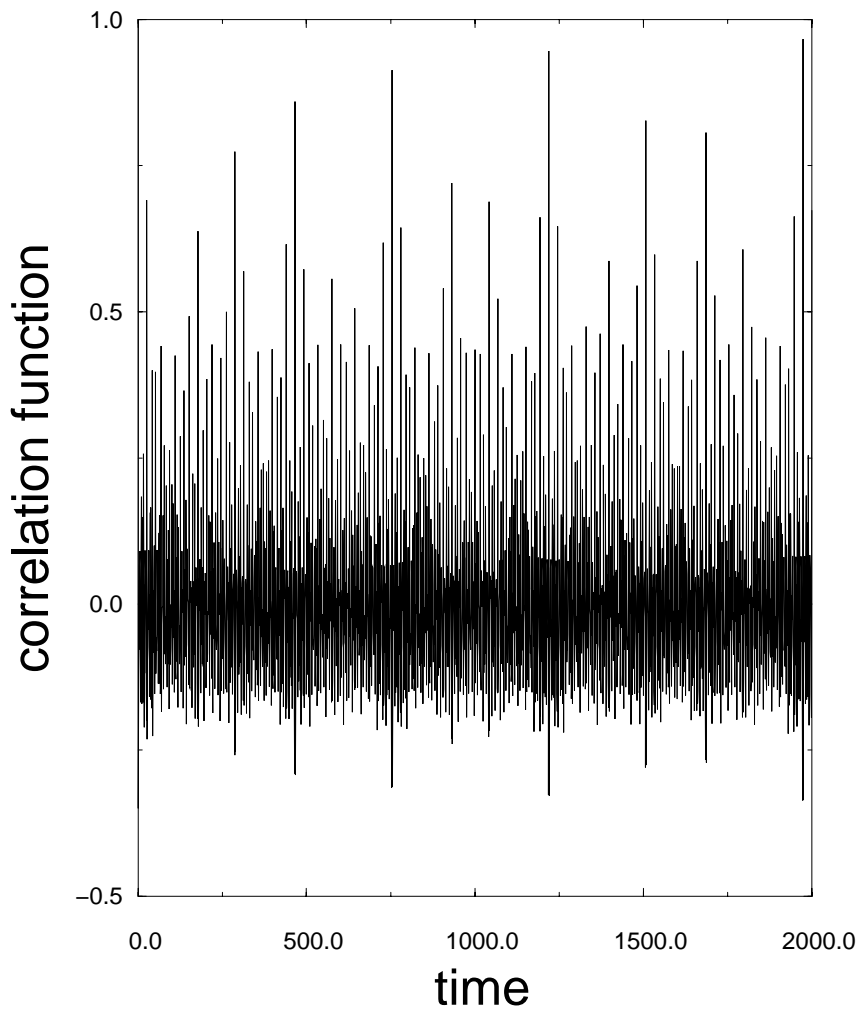

(b)

Fig. 6. Dependence of the correlation functions of the balanced system on the initial conditions of the start position of the items (two panels correspond to two different initial conditions). In both cases the correlation functions come close to one.

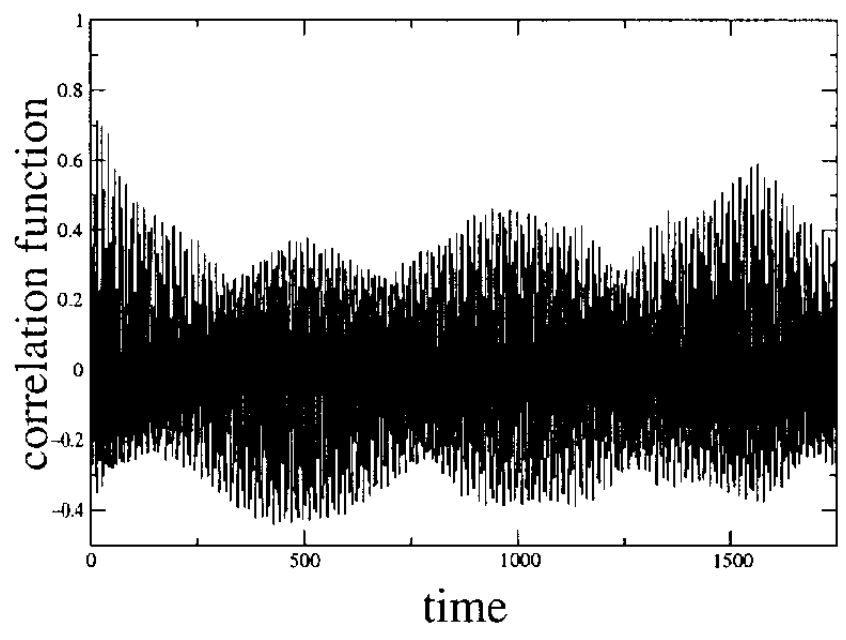

(a)

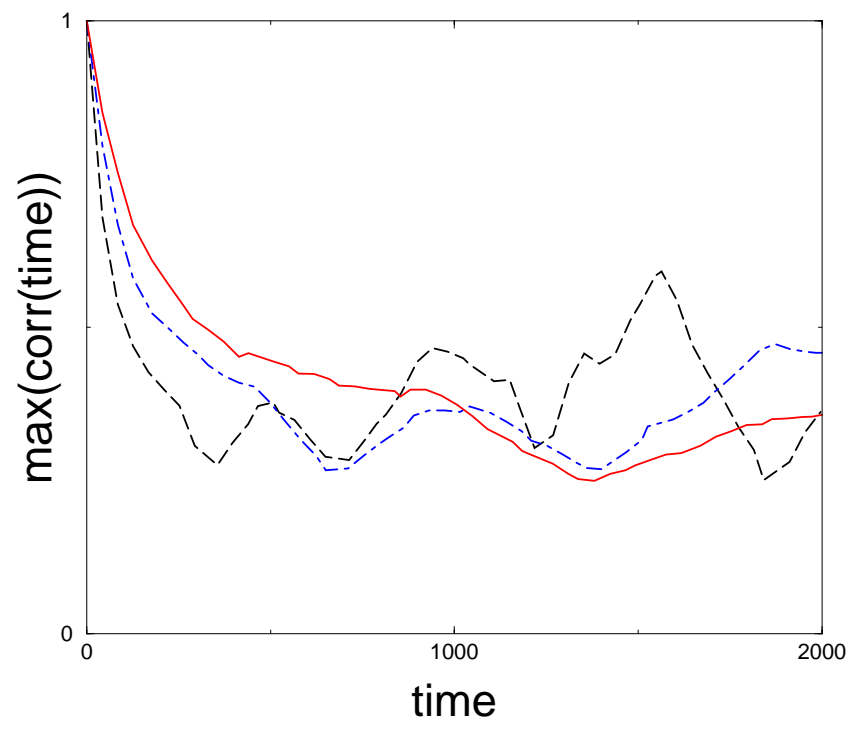

(b)

Fig. 7. (a) Correlation function of an overbalanced system $c=0.98$. (b) The envelope drawn through the peaks of the correlation function. Used parameters: $c=0.98$ (dashed line), $c=0.99$ (dotted-dashed line) and $c=0.995$ (solid line). For all cases the correlation function neither decreases to zero nor returns to one. 


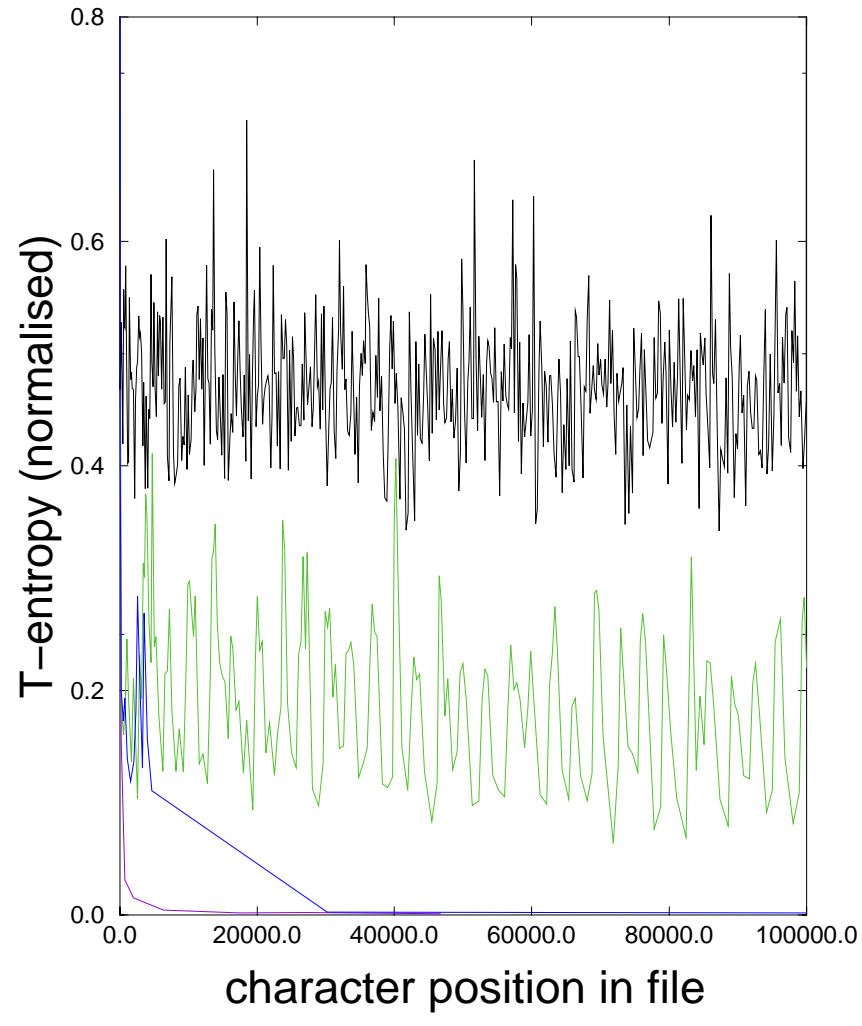

Fig. 8. Estimated T-entropy (normalized by $\log (4)$ ) of different symbolic sequences. From top to bottom: random distributed sequence, $c=0.98, c=1.01$ and balanced system. The sequence consists of four types of symbols and 100000 signs are used.

This method of estimation of the different $k_{n}$ will be repeated until the sequence is identical with the string. With the number of steps $n$ and the estimated $k_{n}$, the T-complexity $C_{T}(x(n))$ of the sequence for the string $x(n)$ is determined as follows:

$$
C_{T}(x(n))=\sum_{n} \log _{N}\left(k_{n}+1\right),
$$

where $N$ is the number of used symbols.

As was shown in [Ebeling et al., 2001], a proper normalization of the T-complexity is needed according to

$$
I_{T}(x(n))=l i^{-1}\left(\frac{C_{T}(x(n))}{\ln N}\right)
$$

where $l i^{-1}$ is the inverse logarithmic integral and the scaling constant is $\ln N$. The quantity $I_{T}(x(n))$ is the T-entropy of the sequence $x(n)$ [Ebeling et al., 2001]. For different sequences the T-entropy is shown in Fig. 8. Because four symbols are used in the investigated sequences, the T-entropy is normalized by $\log (4)$. The T-Entropy of the analyzed sequence has values between the T-Entropy of the quasiperiodic and the random distributed sequence. This confirms the results of the preceding section that the overbalanced system demonstrates behavior more complex than the quasiperiodic one, but nevertheless is not chaotic.

\section{Conclusion}

In this paper we have investigated a simple reentrant model with one machine and two basic types of items to be processed. The main parameter governing the dynamics is the relation between the processing capacity and the delivery times. If the processing capacity is large, the system is underbalanced, and has the properties of a quasiperiodically driven dissipative dynamical system. One can relate this to the fact that for large production rates a queue disappears and the idle time intervals damp out the perturbations. The dissipativity is no more present when the system is balanced: here the queue is never empty and the perturbations do not decay. Moreover, the dynamics depend on the initial state, which can be related to the fact that the perturbations do not grow as well. In the third possible case, when the production capacity is so small that the queue grows continuously, the dynamics becomes more complex than the quasiperiodic one. One can understand this as an effect of the growing queue, which leads to the effective growth of the perturbations. This growth is, however, not fast enough to yield chaos. The correlations in the overbalanced case neither return to one nor decay to zero. A complexity of the queue, characterized with help of the T-entropy, also lies between that of order and noise.

In the paper we have presented the results for one particular set of parameters and the model setup. We have checked also other parameters (e.g. different delivery times and production rates), as well as organization of the queue according to different rules (e.g. when instead of the FIFO rule we gave the top priority to the item $A$, setting it always to the top position in the queue when such an item arrives). In all these situations the qualitative picture was the same as described above, with a maximal complexity in the overbalanced case.

Finally we would like to mention an analogy of the model considered with the models of traffic dynamics [Helbing, 2001]. Consider a situation where two traffic lanes, where cars are driving with different velocities and distances between, have to merge to only one lane (or, in general, the number of lanes is reduced). The merging point represents 
a bottleneck where a traffic jam (in our language a queue) can appear (cf. [Chowdhury \& Schadschneider, 1999; Barlovic et al., 2001; Huisinga et al., 2001]). The underbalanced, balanced, and the overbalanced cases above correspond to a decaying, constant and increasing jam, correspondingly. Contrary to the production dynamics, it is however rather unrealistic to suggest that the cars enter the same bottleneck twice (although in numerical simulations one often takes periodic boundary conditions, so that the outgoing cars simply reenter the queue).

\section{Acknowledgments}

We thank D. Armbruster, M. Zaks, U. Schwarz, M. Freitag and B. Scholz-Reiter for fruitful discussions. I. Katzorke acknowledges support from Volkswagen-Stiftung.

\section{References}

Badii, R. \& Politi, A. [1997] Complexity. Hierarchical Structures and Scaling in Physics (Cambridge University Press, Cambridge).

Barlovic, R., Schadschneider, A. \& Schreckenberg, M. [2001] "Random walk theory of jamming in a cellular automaton model for traffic flow," Physica A294, $525-538$.

Beaumariage, T. \& Kempf, K. [1994] Chaotic Behavior in Manufacturing Systems 2nd Annual Chaos Conf., Santa Fe; "The nature and origin of chaos in manufacturing systems," Proc. 5th IEEE/SEMI Adv. Semiconductor Manufacturing Conf. (Boston), pp. $169-174$.

Bunimovich, L. [2001] "Dynamical systems and operation research: A basic model," Discr. Continuous Dyn. Syst. 1, 209-218.

Chase, C., Serrano, J. \& Ramadge, P. J. [1993] "Periodicity and chaos from switched flow systems: Contrasting examples of discretely controlled continuous systems," IEEE Trans. Automat. Contr. AC-38, p. 70.

Chowdhury, D. \& Schadschneider, A. [1999] "Selforganization of traffic jams in cities: Effects of stochastic dynamics and signal periods," Phys. Rev. E59, 1311-1314.

Diaz-Rivera, I., Armbruster, D. \& Taylor, T. [2000] "Periodic orbits in reentrant manufacturing systems," Math. Oper. Res. 25, 708-725.
Ebeling, W., Steuer, R. \& Titchener, M. R. [2001] "Partition based entropies of deterministic and stochastic maps," Stoch. Dyn. 1, 45-61.

Gross, D. \& Harris, C. M. [1985] Fundamentals of Queueing Theory (Wiley, NY).

Hanson, D., Armbruster, D. \& Taylor, T. [1999] "On the stability of reentrant manufacturing systems," Proc. 31st MTNS, Padua 1998 Mathematical Theory of Networks and Systems, eds. Beghi, A., Finesso, L. \& Picci, G., Il Poligrafo, Padova, Italy, pp. 937-940.

Helbing, D. [2001] "Die wundervolle Welt aktiver Vielteilchensysteme," Physikalische Blaetter 57, 27-33.

Huisinga, T., Barlovic, R., Knospe, W., Schadschneider, A. \& Schreckenberg, M. [2001] "A microscopic model for packet transport in the Internet," Physica A294, 249-256.

Katzorke, I. \& Pikovsky, A. [2000] Chaos and Complexity in Simple Models of Production Dynamics (Discrete Dynamics in Nature and Society), pp. 179-187.

Nelson, R. [1995] Probability, Stochastic Processes, and Queueing Theory (Springer, NY).

Pikovsky, A. \& Feudel, U. [1994] "Correlations and spectra of strange nonchaotic attractors," J. Phys. A27, 5209-5219.

Press, W. H., Flannery, B. P., Teukolsky, S. A. \& Vetterling, W. T. [1989] Numerical Recipes (Cambridge University Press, Cambridge).

Queffélec, M. [1987] Substitution Dynamical Systems Spectral Analysis, Springer Lecture Notes in Mathematics, Vol. 1294, Berlin.

Titchener, M. R. [1994] A Model for String Complexity, Salodays in Auckland Proc., pp. 101-105.

Titchener, M. R. [1998] A Novel Deterministic Approach to Evaluating the Entropy of Language Texts, Itallc, Hsittou, Taiwan.

Titchener, M. R. [2001] "A measure of information," Proc. Data Compression Conf. (DCC '00), Snowbird, Utah (IEEE), pp. 353-362.

Schürmann, T. \& Hoffmann, I. [1995] "The entropy of 'strange billiards' inside $n$-simplexes," J. Phys. A28, 5033-5039.

Zaks, M., Pikovsky, A. S. \& Kurths, J. [1998] "Symbolic dynamics behind the singular continuous power spectra of continuous flow," Physica D117, 77-94.

Zaks, M. [2001] "Fractal Fourier spectra of Cherry flow," Physica D149, 237-247. 